\title{
Testing Substitutability
}

\author{
John William Hatfield \\ Graduate School of Business, Stanford University \\ Nicole Immorlica \\ McCormick School of Engineering and Kellogg Graduate School of Management, \\ Northwestern University \\ Scott Duke Kominers ${ }^{* 1}$ \\ Becker Friedman Institute for Research in Economics, University of Chicago
}

\begin{abstract}
We provide an algorithm for testing the substitutability of a length- $N$ preference relation over a set of contracts $X$ in time $O\left(|X|^{3} \cdot N^{3}\right)$. Access to the preference relation is essential for this result: We show that a substitutability-testing algorithm with access only to an agent's choice function must make an expected number of queries exponential in $|X|$. An analogous result obtains when the agent's preferences are quasilinear in a numeraire and the algorithm has access to the agent's underlying valuation function.
\end{abstract}

Key words: substitutability, matching, communication complexity, preference elicitation.

JEL: C62, C78

\section{Introduction}

The theory of two-sided matching has been widely applied in medical labor markets such as the National Resident Matching Program (Roth and Peranson (1999)), the redesign of the gastroenterology fellowship market (Niederle and Roth (2003, 2005); McKinney et al. (2005)), and the job market for clinical psychologists (Roth and Xing (1997)). ${ }^{2}$ The centralized matching mechanisms

\footnotetext{
* Corresponding author.

Email addresses: hatfield@stanford.edu (John William Hatfield), nickle@eecs.northwestern.edu (Nicole Immorlica), skominers@uchicago.edu (Scott Duke Kominers), skominers@gmail.com (Scott Duke Kominers)

${ }^{1}$ Department of Economics, University of Chicago, 1126 East 59th Street, Chicago, IL 60637.

${ }^{2}$ Roth and Sotomayor (1990) and Roth (2008) survey the theory and practice of matching market design.
}

Preprint submitted to Games and Economic Behavior

December 3, 2011 
used in these markets require that hospitals submit responsive preferences over doctors, that is, preferences that render the choice between a pair of doctors independent of other available outcomes. ${ }^{3}$ Although responsive preferences are easy to specify, they are unnatural in many settings: for instance, if a hospital desires both a cardiologist and a radiologist - and doctors of the same specialty substitute for one another - then that hospital's preferences are not responsive.

An alternative would be to allow hospitals to submit preferences that encode substitutabilities between doctors, such as those described above. This is possible within the domain of substitutable preferences introduced by Roth (1984); this domain is of particular interest, as in many settings it is the largest domain for which stable matching outcomes can be guaranteed. ${ }^{4}$ For the implementation of matching mechanisms with substitutable preferences, it is necessary that the market designer have a test which determines if a hospital's submitted preference list is substitutable. In this paper, we provide such a test. While a naïve test requires time exponential in the number of options available to each hospital, we obtain a deterministic substitutability-testing algorithm that runs in time polynomial in the number of options and the preference list length.

Unfortunately, as we demonstrate, the efficient testing of substitutability depends crucially on the form in which agents' preferences are submitted. In particular, we show that when the market designer only has oracle access to hospitals' choice functions, substitutability can not be tested in subexponential time - even by randomized algorithms.

Classical matching theory typically assumes that agents only submit ordinal preference information, such as preference relations or choice functions. ${ }^{5}$ However, it is often appropriate to consider instead cardinal utility information, as in auction theory or exchange economies. ${ }^{6}$ Unfortunately, we find that the difficulty of testing substitutability given only choice function access carries over to the setting where the market designer can query valuation functions.

The remainder of this paper is organized as follows. In Section 2, we present our algorithm for testing the substitutability of a preference relation. In Sections 3 and 4 , we show the difficulty of testing the substitutability of choice and

\footnotetext{
${ }^{3}$ More formally, a hospital's preference relation is responsive if it is consistent with an ordinal ranking of doctors.

${ }^{4}$ Substitutability is necessary and sufficient for the existence of stable allocations in the settings of many-to-one matching (Roth (1984) proves sufficiency and Hatfield and Kojima (2008) prove necessity), many-to-many matching, (Roth (1984) and Echenique and Oviedo (2006) prove sufficiency and necessity follows from the results of Hatfield and Kojima (2008)), many-to-many matching with contracts (Klaus and Walzl (2009) and Hatfield and Kominers (2011) prove sufficiency and Hatfield and Kominers (2011) prove necessity), and supply-chain matching (Ostrovsky (2008) and Hatfield and Kominers (forthcoming) prove sufficiency and Hatfield and Kominers (forthcoming) prove necessity). In the setting of many-to-one matching with contracts, substitutable preferences are sufficient for the existence of a stable outcome (Hatfield and Milgrom (2005)), but are not necessary (Hatfield and Kojima (2008, 2010)).

${ }^{5}$ This is a standard assumption in many applications of matching, such as school choice, where monetary transfers among market participants are not allowed.

${ }^{6}$ As in matching theory, substitutability is essential in those settings. See, in particular, the work of Gul and Stacchetti (1999), Sun and Yang (2006), and Hatfield et al. (2011).
} 
valuation functions, respectively. Section 5 concludes.

\section{Testing Substitutability of Preference Relations}

We consider the preferences of a single agent and a finite set $X$ of contracts available to that agent. The agent's preferences are specified by a preference relation $\succ$ of the form

$$
Y^{1} \succ Y^{2} \succ \cdots \succ Y^{N} \succ \varnothing
$$

where $Y^{n} \subseteq X$ for each $n=1, \ldots, N$. This preference relation induces a choice function

$$
C(Y) \equiv \max _{\succ}\{Z: Z \subseteq Y\}
$$

on every set of contracts $Y \subseteq X{ }^{7}$ Note that we suppress the dependence on the preference relation $\succ$ in the notation for $C$, as throughout this section we will only consider a fixed preference relation $\succ$ and its associated choice function.

Definition. The preference relation $\succ$ is substitutable if for all $x, z \in X$ and $Y \subseteq X$, if $z \in C(\{x\} \cup Y \cup\{z\})$, then $z \in C(Y \cup\{z\})$. A tuple $(x, z, Y)$ that fails this condition is called a substitutability violation.

Equivalently, if we define a rejection function

$$
R(Y) \equiv Y \backslash C(Y),
$$

then the preference relation $\succ$ is substitutable if and only if the rejection function $R$ is isotone. ${ }^{8}$ Intuitively, this notion implies that no contracts $x$ and $z$ are complements: that is, substitutability guarantees that, having rejected the contract $z$, the agent never wishes to renege on that rejection when more contracts become available.

As stated, the substitutability condition imposes exponentially many conditions on the preference relation: one condition for each tuple $(x, z, Y)$. Thus, naïve substitutability-testing takes $O\left(|X|^{3} \cdot 2^{|X|}\right)$ time. Here we present an algorithm that tests preference relation substitutability in time polynomial in $|X|$, the number of contracts, and $N$, (defined as) the length of the submitted preference list. ${ }^{9}$

Intuitively, since the agent only chooses contract sets appearing in the preference list $Y^{1} \succ \cdots \succ Y^{N} \succ \varnothing$, one might think it only necessary to check for

\footnotetext{
${ }^{7}$ Here, we use the notation $\max \succ$ to indicate that the maximization is taken with respect to $\succ$.

${ }^{8}$ Isotonicity of the rejection function can be stated in a fashion that makes substitutability violations apparent: $R$ is isotone (equivalently, $C$ is substitutable) if and only if for all $x, z \in X$, $x \neq z$, and $Y \subseteq X$, if $z \in C(Y)$, then $z \in C(Y \backslash\{x\})$.

${ }^{9}$ Note that in some circumstances, $N$ may be $O\left(2^{|X|}\right)$, in which case our algorithm runs in time of the same order as that of the naïve algorithm. However, in many cases (such as when hospitals only intend to hire some small number of doctors from a large pool), our algorithm provides a significant improvement-a polynomial run time.
} 
the violation of substitutability by tuples $\left(x, z, Y^{n}\right)$ with $x, z \in \cup_{m} Y^{m}$. In fact this is not sufficient, as the following example shows.

Example 1. Let $X=\left\{a, b, b^{\prime}, c\right\}$ and let the preference relation be

$$
\{a, b, c\} \succ\left\{b^{\prime}\right\} \succ\{a, b\} \succ\{a, c\} \succ\{b, c\} \succ\{a\} \succ\{b\} \succ\{c\} \succ \varnothing .
$$

These preferences are not substitutable, as $\left(a, c,\left\{b, b^{\prime}\right\}\right)$ is a substitutability violation. However, no substitutability violation is of the form $\left(x, z, Y^{n}\right)$.

While it is sufficient to consider only $(x, z, Y)$ with $x, z \in \cup_{m} Y^{m}$, it is not sufficient to check only $Y=Y^{n}$, as $Y$ may need to contain multiple contracts that will only be chosen when another contract $(x)$ is available. However, the intuition that one need only check sets $Y$ derived from the sets $Y^{n}$ is sound. In particular, as we show below, it suffices to check all tuples of the form $\left(x, z,\left(Y^{m} \cup Y^{n}\right) \backslash\{x, z\}\right)$. This gives rise to Algorithm 1, which in fact returns a list of all minimal substitutability violations, i.e. all violating tuples $(x, z, Y)$ for which there does not exist $Y^{\prime} \subsetneq Y$ such that $\left(x, z, Y^{\prime}\right)$ is a substitutability violation.

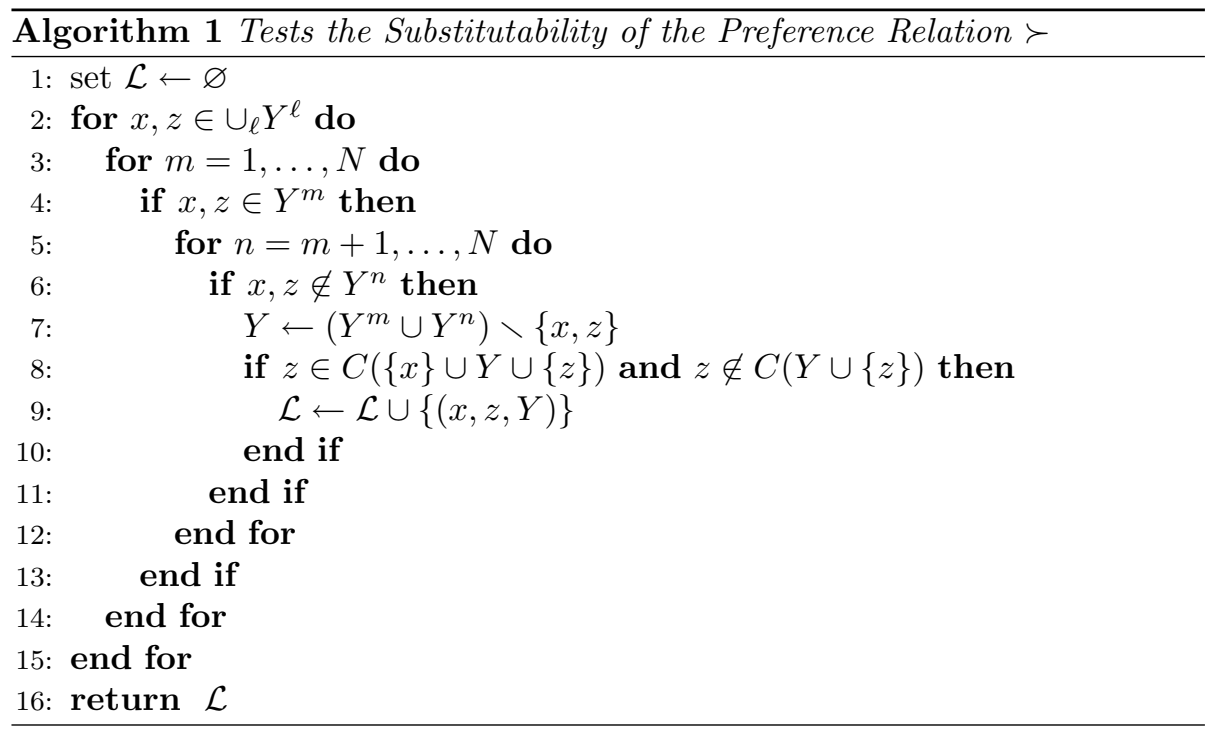

Algorithm 1 finds the (minimal) substitutability violation in Example 1 when checking contracts $a$ and $c$ in line 2. At that point, when $m=1$ and $n=2$, $Y^{m}=\{a, b, c\}$ and $Y^{n}=\left\{b^{\prime}\right\}$, and the tuple $(x, z, Y)$ is $\left(a, c,\left\{b, b^{\prime}\right\}\right)$.

To prove the efficacy of Algorithm 1, we make repeated use of the fact that the choice function $C$ induced by $\succ$ has the property that removing unchosen contracts from the available set has no effect on the set of contracts chosen. ${ }^{10}$

\footnotetext{
${ }^{10}$ This is analogous to the notion of independence of irrelevant alternatives in revealed preference theory.
} 
Lemma 1. For the choice function $C$ induced by the preference relation $Y^{1} \succ$ $Y^{2} \succ \cdots \succ Y^{N} \succ \varnothing$, let $n$ be such that $Y^{n}=C(Y)$ for $Y \subseteq X$. Then for any $Y^{\prime} \subseteq Y$ such that $Y^{n} \subseteq Y^{\prime}$, we have $Y^{n}=C\left(Y^{\prime}\right)$.

Proof. Suppose $Y^{m}=C\left(Y^{\prime}\right)$. If $m<n$, then since $Y^{m}$ is available from $Y$, it can not be the case that $Y^{n}=C(Y)$. We can not have that $m>n$, as $Y^{n}$ is available from $Y^{\prime}$.

We now prove the correctness of Algorithm 1.

Theorem 1. Algorithm 1 finds all minimal substitutability violations in a preference relation $Y^{1} \succ \cdots \succ Y^{N} \succ \varnothing$ in time $O\left(|X|^{3} \cdot N^{3}\right)$.

Proof. The time bounds are immediate from the statement of the algorithm, upon noting that computing $C(Z)$ for any $Z \subseteq X$ requires at most $O(|X| \cdot N)$ time. ${ }^{11}$ To show correctness, suppose there exists a set $\hat{W}$ such that the tuple $(x, z, \hat{W})$ is a substitutability violation. Letting $W=\hat{W} \backslash\{x, z\}$, we see that $(x, z, W)$ is a subsitutability violation as well, and if $(x, z, \hat{W})$ is minimal, then $W=\hat{W}$. Let $Y^{m}=C(W \cup\{x, z\})$ be the choice from options $W \cup\{x, z\}$ and $Y^{n}=C(W \cup\{z\})$ be the choice from options $W \cup\{z\} .{ }^{12}$ Set $Y=\left(Y^{m} \cup Y^{n}\right) \backslash$ $\{x, z\}$. We will show that the tuple $(x, z, Y)$ is a substitutability violation.

- First note that as $(x, z, W)$ is a substitutability violation, $z \in Y^{m}=$ $C(W \cup\{x, z\})$. Furthermore, $Y \cup\{x, z\} \subseteq W \cup\{x, z\}$ and $C(W \cup\{x, z\})=$ $Y^{m} \subseteq Y \cup\{x, z\}$ so, by Lemma $1, C(Y \cup\{x, z\})=C(W \cup\{x, z\})=Y^{m}$. Thus, $z \in C(Y \cup\{x, z\})$.

- Next note that as $(x, z, W)$ is a substitutability violation, we also have $z \notin Y^{n}=C(Y \cup\{z\})$. Again, $Y \cup\{z\} \subseteq W \cup\{z\}$ and $C(W \cup\{z\})=$ $Y^{n} \subseteq Y \cup\{z\}$ so, by Lemma $1, C(Y \cup\{z\})=C(W \cup\{z\})=Y^{n}$. Thus, $z \notin C(Y \cup\{z\})$.

This shows that $(x, z, Y)$ is a substitutability violation.

To see that the algorithm finds all minimal violations, simply note that for any substitutability violation $(x, z, W)$, we know that for the returned tuple $(x, z, Y)$, we have that $Y \subseteq W$ and $(x, z, Y)$ is a violation. Thus if $(x, z, W)$ is minimal, it must be that $Y=W$.

Given the list of minimal substitutability violations, it is easy to check (in constant time for each violation) whether these violations constitute violations of the weaker bilateral and unilateral substitutes conditions introduced by Hatfield and Kojima (2010). Thus, Algorithm 1 can easily be extended to test for bilateral or unilateral substitutability in time polynomial in $|X|$ and $N$.

\footnotetext{
${ }^{11}$ Note that calculating $C(Z)$ requires us to check whether $Z$ is a subset of $Y^{n}$ for $n=$ $1, \ldots, N$ and that checking the subset relation requires $O(|X|)$ time; hence, calculating $C(Z)$ requires $O(|X| \cdot N)$ time.

${ }^{12}$ Note that $x, z \notin Y^{n}$, as $x, z \notin W$ and $(x, z, W)$ is a substitutability violation. Thus, without loss of functionality (and with a slight gain in speed) we may include line 6 in Algorithm 1.
} 


\section{Testing Substitutability of Choice Functions}

We now consider algorithms which have oracle access to the agent's choice function over contracts, $C$, but do not have access to the preference relation itself. We show that no algorithm can verify the choice function's substitutability in subexponential time. The key observation underlying this result is that a substitutability violation is "local" - it is possible for a choice function to have very few substitutability violations relative to the number of sets of contracts.

Our proof uses the minimax principle of Yao (1977), which states that the expected running time of the best randomized algorithm on a worst-case deterministic input is equal to the expected running time of the best deterministic algorithm on the worst-case probability distribution of inputs. ${ }^{13}$

More precisely, for a problem with deterministic inputs $\mathcal{I}$ and deterministic algorithms $\mathcal{A}$, let $\Delta(\mathcal{I})$ denote the set of probability distributions over $\mathcal{I}$ and $\Delta(\mathcal{A})$ denote the set of probability distributions over $\mathcal{A}$. For $A \in \mathcal{A}$ and $I \in \mathcal{I}$, let $A(I)$ denote the running time of $A$ on $I$.

Theorem 2 (Yao (1977)). For a problem with deterministic inputs $\mathcal{I}$ and deterministic algorithms $\mathcal{A}$,

$$
\min _{\delta_{\mathcal{A}} \in \Delta(\mathcal{A})} \max _{I \in \mathcal{I}} E_{A \sim \delta_{\mathcal{A}}}[A(I)]=\max _{\delta_{\mathcal{I}} \in \Delta(\mathcal{I})} \min _{A \in \mathcal{A}} E_{I \sim \delta_{\mathcal{I}}}[A(I)]
$$

The proof of Theorem 2 follows from von Neumann's minimax theorem for two-player, zero-sum games. Here, the two players are the algorithm designer and the adversary. The algorithm designer's strategy set is all possible deterministic algorithms $\mathcal{A}$; the adversary's strategy set is all possible deterministic inputs $\mathcal{I}$. The payoff of a pure strategy profile $(A, I)$ to the adversary is the running time $A(I)$ (the payoff to the algorithm designer is the negative of this, as we are defining a zero-sum game). For further details, we refer the reader to Yao (1977).

We now apply Theorem 2 to show that a substitutability-testing algorithm with only oracle access to $C$ must make exponentially many queries in expectation. For any $Y \subsetneq X$ with $|X \backslash Y| \geq 2$, we define the following choice function (implicitly assuming $|X|>3$ ):

$$
C_{Y}(Z)= \begin{cases}Y & Y \subseteq Z \text { and }|Z|=|Y|+1, \\ Z & \text { otherwise. }\end{cases}
$$

We note that $C_{Y}$ is not substitutable: for any $x, z \notin Y$, we have $z \notin Y=$ $C_{Y}(Y \cup\{z\})$, but $z \in\{x\} \cup Y \cup\{z\}=C_{Y}(\{x\} \cup Y \cup\{z\})$.

Now we consider any deterministic substitutability testing algorithm $A$ and suppose that the distribution of inputs is as follows. With probability $\frac{1}{2}$, set $C=$ id, where id is the identity function on $X$, i.e. $\operatorname{id}(Z)=Z$ for all $Z \subseteq X$.

\footnotetext{
${ }^{13}$ Here, by a randomized algorithm, we mean a probability distribution over deterministic algorithms.
} 
With the remaining probability, select a set $Y$ of cardinality $q \equiv\left\lfloor\frac{|X|}{2}\right\rfloor$ uniformly at random and set $C=C_{Y}$.

We observe that, conditional on $C=\mathrm{id}, A$ must query at least

$$
\frac{1}{q+1}\left(\begin{array}{c}
|X| \\
q
\end{array}\right)
$$

sets in order to be sure that no substitutabilility violation exists: Since the functions id and $C_{Y}$ only differ on input sets $Z \supsetneq Y$ of size $|Z|=|Y|+1$, only queries on such sets $Z$ can distinguish them. Thus, verifying that $C=$ id requires distinguishing $C$ from $\left(\begin{array}{c}|X| \\ q\end{array}\right)$ possible assignments of $C_{Y}$. Querying $C$ on a given set $Z$ of size $q+1$ simultaneously tests whether $C=C_{Z \backslash\{z\}}$ for each $z \in Z$, checking a total of $q+1$ possible assignments of $C_{Y}$. Hence, when $C=\mathrm{id}$, the algorithm $A$ must check at least as many sets of size $q+1$ as in (1). Since $C=$ id with probability $\frac{1}{2}$, the expected running time must be at least $\frac{1}{2}$ times the bound in formula (1). ${ }^{14}$

Theorem 3. There exists a distribution of choice functions $\delta$ such that for any deterministic algorithm $A$ that verifies substitutability given only oracle access to the choice function, the expected running time of A given $\delta$ is at least exponential in $|X|$, the number of contracts.

Combining Theorems 2 and 3, we obtain the following corollary.

Corollary 1. The worst-case expected running time of any randomized algorithm that verifies substitutability given only oracle access to choice functions is at least exponential in $|X|$, the number of contracts.

We note that even allowing polynomial error in the algorithm does not improve the running time substantially, i.e. there is no subexponential-time algorithm that verifies substitutability correctly with probability $1-o(1)$. Additionally, it follows from the above argument that testing responsiveness is as difficult as testing substitutability when given only oracle access to the choice function. ${ }^{15}$

\section{Testing Substitutability of Valuation Functions}

We now consider the case where each contract defines the transfer of an item at an associated price; let the set of items be denoted $\Omega$, and let the price of $\omega \in \Omega$ be denoted $p_{\omega}$. For many applications (e.g., Kelso and Crawford (1982);

\footnotetext{
${ }^{14}$ Unfortunately, the choice functions used in this proof do not have preference relation encodings of length polynomial in $|X|$. This implies that Algorithm 1 cannot verify the substitutability of these preferences in time polynomial in $|X|$. This leaves open the question of whether there exist preferences for which substitutability can be verified in polynomial time using the preference relation, but only in exponential time given oracle access to the choice function.

${ }^{15}$ We thank Assaf Romm for this observation.
} 
Gul and Stacchetti (1999, 2000); Sun and Yang (2006, 2009); Hatfield et al. (2011)), it is assumed that agents' utility functions take the quasilinear form

$$
U(\Psi, p)=v(\Psi)-\sum_{\psi \in \Psi} p_{\psi},
$$

for $\Psi \subseteq \Omega$.

From here, we can define the demand correspondence $D: \mathbb{R}^{|\Omega|} \rightarrow \mathcal{P}(\Omega)$,

$$
D(p) \equiv \underset{\Psi \subseteq \Omega}{\arg \max } U(\Psi, p) .
$$

Kelso and Crawford (1982) introduced the following natural notion of substitutability, which has been shown to be essential for the guaranteed existence of stable allocations and competitive equilibria in a variety of settings (Hatfield et al. $(2011)){ }^{16}$

Definition. A demand correspondence $D$ satisfies the gross substitutes condition if for all profiles of prices $p$ and $\hat{p}$ such that $\hat{p} \geq p$, for every $\Psi \in D(p)$ there exists a $\hat{\Psi} \in D(\hat{p})$ such that $\left\{\omega \in \Psi: p_{\omega}=\hat{p}_{\omega}\right\} \subseteq \hat{\Psi}$.

The gross substitutes condition has been rephrased in terms of conditions on the valuation function $v$.

Theorem 4 (Reijnierse et al. (2002); Fujishige and Yang (2003)). A demand correpondence $D$ satisfies the gross substitutes condition if and only if the corresponding valuation function $v$ is $M^{\natural}$-concave, i.e. if for each $\Psi, \Phi \subseteq \Omega$ and $\psi \in \Psi \backslash \Phi:$

1. $v(\Psi)+v(\Phi) \leq v(\Psi \backslash\{\psi\})+v(\Phi \cup\{\psi\})$, and

2. $v(\Psi)+v(\Phi) \leq \max _{\varphi \in \Phi \backslash \Psi} v((\Psi \backslash\{\psi\}) \cup\{\varphi\})+v((\Phi \backslash\{\varphi\}) \cup\{\psi\})$.

As Reijnierse et al. (2002) point out, this theorem implies an algorithm that tests gross substitutability and runs in time $O\left(|X|^{3} \cdot 2^{|X|}\right)$. We now show that this algorithm is optimal up to polynomial factors; i.e. there is no subexponential-time algorithm that verifies gross substitutability given oracle access to the valuation function. ${ }^{17}$

Theorem 5. There exists a distribution of valuations $\delta$ such that for any deterministic algorithm $A$ that verifies substitutability given only oracle access to the valuation, the expected running time of $A$ given $\delta$ is at least exponential in $|\Omega|$, the number of items.

\footnotetext{
${ }^{16}$ While the definition presented here is standard, there are many equivalent formulations; see Hatfield et al. (2011) for a discussion.

${ }^{17}$ This result is not an exact analogue of Theorem 3 , as it involves queries to valuation functions rather than demand correspondences. The complexity of testing gross substitutability given oracle access to the demand correspondence is presently unclear. The difficulty involved in proving an exact analogue of Theorem 3 arises from the fact that demand correspondences are functions of prices, rather than sets of items.
} 
Corollary 2. The worst-case expected running time of any randomized algorithm that verifies substitutability given only oracle access to valuations is at least exponential in $|\Omega|$, the number of items.

The proof of these results follows the same strategy as that used to prove Theorem 3 and Corollary 1. For any $\Psi \subsetneq \Omega$ with $|\Omega \backslash \Psi| \geq 2$, we define the following valuation function:

$$
v_{\Psi}(\Phi)= \begin{cases}|\Phi|+\frac{1}{2} & \Phi=\Psi \\ |\Phi| & \text { otherwise }\end{cases}
$$

Note that $v_{\Psi}$ is not $M^{\natural}$-concave since for any $\psi \in \Psi$, as

$$
v_{\Psi}(\Psi)+v_{\Psi}(\emptyset)=|\Psi|+\frac{1}{2}
$$

and

$$
v_{\Psi}(\Psi \backslash\{\psi\})+v_{\Psi}(\{\psi\})=|\Psi|-1+1=|\Psi|,
$$

and so the first condition of $M^{\natural}$-concavity is violated.

As in the proof of Theorem 3, distinguishing $v_{\Psi}$ from the valuation $\hat{v}$ defined by $\hat{v}(\Phi)=|\Phi|$ requires exponentially many queries in expectation. Moreover, as in the case of Theorem 3 and Corollary 1, allowing polynomial error in the algorithm does not improve the running time significantly.

\section{Conclusion}

We have shown a method for testing the substitutability of an agent's preferences relations in time polynomial in the length of the preference relation. Since agents are, in general, only able to construct short (i.e. polynomial length) preferences lists, our algorithm is an effective method for the market designer to test whether the submitted preferences are substitutable. In principle, such an algorithm could be distributed to market participants for use in the preperation of their preference relations for submission.

Unfortunately, testing substitutability with only oracle access to the choice function is far more difficult - it requires exponential time in general. The problem persists even for the special case in which preferences are quasilinear in a numeraire and only valuation function access is available. We note however that as the choice functions used in this proof do not have preference relation encodings of length polynomial in $|X|$, it remains unknown whether there exist preferences for which substitutability can be verified in polynomial time using the preference relation, but only in exponential time given oracle access to the choice function.

Our results imply that market designers should develop specific methods to transmit large classes of substitutable preferences. Milgrom (2009) provides one such method, assignment messages, for enviroments such as electricity and commodity markets where preferences are quasilinear. For other settings, it 
is likely that alternative languages for expressing substitutable preferences are needed. It is unclear how general such a language can be while remaining effective, but our results do not rule out the existence of an encoding under which all substitutable preferences take a form of at most polynomial size.

\section{Acknowledgments}

We thank Assaf Romm, Alvin E. Roth, and the referees for helpful comments and suggestions. Hatfield appreciates the hospitality of Harvard Business School, which hosted him during parts of this research. Kominers gratefully acknowledges the support of a National Science Foundation Graduate Research Fellowship, a Yahoo! Key Scientific Challenges Program Fellowship, and a Terence M. Considine Fellowship in Law and Economics funded by the John M. Olin Center at Harvard Law School.

\section{References}

Echenique, F., Oviedo, J., 2006. A theory of stability in many-to-many matching markets. Theoretical Economics 1, 233-273.

Fujishige, S., Yang, Z., 2003. A note on Kelso and Crawford's gross substitutes condition. Mathematics of Operations Research, 463-469.

Gul, F., Stacchetti, E., 1999. Walrasian equilibrium with gross substitutes. Journal of Economic Theory 87, 95-124.

Gul, F., Stacchetti, E., 2000. The English auction with differentiated commodities. Journal of Economic Theory 92, 66-95.

Hatfield, J. W., Kojima, F., 2008. Matching with contracts: Comment. American Economic Review 98, 1189-1194.

Hatfield, J. W., Kojima, F., 2010. Substitutes and stability for matching with contracts. Journal of Economic Theory 145 (5), 1704-1723.

Hatfield, J. W., Kominers, S. D., 2011. Contract design and stability in matching markets, Mimeo, Harvard Business School.

Hatfield, J. W., Kominers, S. D., forthcoming. Matching in Networks with Bilateral Contracts. American Economic Journal: Microeconomics.

Hatfield, J. W., Kominers, S. D., Nichifor, A., Ostrovsky, M., Westkamp, A., 2011. Stability and competitive equilibrium in trading networks, mimeo, Stanford Graduate School of Business.

Hatfield, J. W., Milgrom, P., 2005. Matching with contracts. American Economic Review 95, 913-935. 
Kelso, A. S., Crawford, V. P., 1982. Job matching, coalition formation, and gross substitutes. Econometrica 50, 1483-1504.

Klaus, B., Walzl, M., 2009. Stable many-to-many matchings with contracts. Journal of Mathematical Economics 45 (7-8), 422-434.

McKinney, C. N., Niederle, M., Roth, A. E., 2005. The collapse of a medical labor clearinghouse (and why such failures are rare). American Economic Review 95, 878-889.

Milgrom, P., 2009. Assignment messages and exchanges. American Economic Journal: Microeconomics 1, 95-113.

Niederle, M., Roth, A. E., 2003. Unraveling reduces mobility in a labor market: Gastroenterology with and without a centralized match. Journal of Political Economy 111, 1342-1352.

Niederle, M., Roth, A. E., 2005. The gastroenterology fellowship market: Should there be a match? American Economic Review 95, 372-375.

Ostrovsky, M., 2008. Stability in supply chain networks. American Economic Review 98, 897-923.

Reijnierse, H., van Gellekom, A., Potters, J. A. M., 2002. Verifying gross substitutability. Economic Theory 20 (4), 767-776.

Roth, A. E., 1984. Stability and polarization of interests in job matching. Econometrica $52,47-57$.

Roth, A. E., 2008. Deferred acceptance algorithms: history, theory, practice, and open questions. International Journal of Game Theory 36, 537-569.

Roth, A. E., Peranson, E., 1999. The effects of the change in the NRMP matching algorithm. American Economic Review 89, 748-780.

Roth, A. E., Sotomayor, M. A. O., 1990. Two-sided matching: a study in gametheoretic modeling and analysis. Cambridge University Press.

Roth, A. E., Xing, X., 1997. Turnaround time and bottlenecks in market clearing: Decentralized matching in the market for clinical psychologists. Journal of Political Economy 105, 284-329.

Sun, N., Yang, Z., 2006. Equilibria and indivisibilities: gross substitutes and complements. Econometrica 74, 1385-1402.

Sun, N., Yang, Z., 2009. A double-track adjustment process for discrete markets with substitutes and complements. Econometrica 77, 933-952.

Yao, A. C.-C., 1977. Probabilistic computations: Toward a unified measure of complexity. In: Proceedings of the 18th Annual Symposium on Foundations of Computer Science (FOCS). pp. 222-227. 